\title{
Interatomic potential for silicon defects and disordered phases
}

\section{Citation}

Justo, João F., Martin Z. Bazant, Efthimios Kaxiras, V. V. Bulatov, and Sidney Yip. 1998. "Interatomic Potential for Silicon Defects and Disordered Phases." Physical Review B 58 (5): 2539-50. https://doi.org/10.1103/physrevb.58.2539.

\section{Permanent link}

http://nrs.harvard.edu/urn-3:HUL.InstRepos:41384076

\section{Terms of Use}

This article was downloaded from Harvard University's DASH repository, and is made available under the terms and conditions applicable to Other Posted Material, as set forth at http:// nrs.harvard.edu/urn-3:HUL.InstRepos:dash.current.terms-of-use\#LAA

\section{Share Your Story}

The Harvard community has made this article openly available.

Please share how this access benefits you. Submit a story.

Accessibility 


\title{
Interatomic Potential for Silicon Defects and Disordered Phases
}

\author{
João F. Justo( ${ }^{(1)}$, Martin Z. Bazant ${ }^{(2)}$, Efthimios Kaxiras ${ }^{(2)}$, V. V. Bulatov ${ }^{(3)}$, and Sidney Yip ${ }^{(4)}$ \\ ${ }^{(1)}$ Instituto de Física da Universidade de São Paulo, CP 66318, CEP 05315-970 São Paulo - SP, Brazil \\ ${ }^{(2)}$ Department of Physics, Harvard University, Cambridge, MA 02138 \\ (3) Department of Mechanical Engineering, Massachusetts Institute of Technology, Cambridge, MA 02139 \\ ${ }^{(4)}$ Department of Nuclear Engineering, Massachusetts Institute of Technology, Cambridge, MA 02139
}

(December 1, 1997)

\begin{abstract}
We develop an empirical potential for silicon which represents a considerable improvement over existing models in describing local bonding for bulk defects and disordered phases. The model consists of two- and three-body interactions with theoretically motivated functional forms that capture chemical and physical trends as explained in a companion paper. The numerical parameters in the functional form are obtained by fitting to a set of $a b$ initio results from quantum mechanical calculations based on density functional theory in the local density approximation, which include various bulk phases and defect structures. We test the potential by applying it to the relaxation of point defects, core properties of partial dislocations and the structure of disordered phases, none of which are included in the fitting procedure. For dislocations, our model makes predictions in excellent agreement with $a b$ initio and tight-binding calculations. It is the only potential known to describe both the $30^{\circ}$ - and $90^{\circ}$-partial dislocations in the glide set $\{111\}$. The structural and thermodynamic properties of the liquid and amorphous phases are also in good agreement with experimental and $a b$ initio results. Our potential is the first capable of simulating a quench directly from the liquid to the amorphous phase, and the resulting amorphous structure is more realistic than with existing empirical preparation methods. These advances in transferability come with no extra computational cost, since force evaluation with our model is faster than with the popular potential of Stillinger-Weber, thus allowing reliable atomistic simulations of very large atomic systems.
\end{abstract}

\section{INTRODUCTION}

Silicon is one of the most intensely investigated materials. Interest in silicon is mostly motivated by its great technological importance, but it is also regarded as the prototypical covalent solid, on which theoretical concepts about covalent bonding can be tested, and new ideas can be explored. The nature of covalent bonding often makes the description of complicated phenomena difficult: the covalent bond can only be described properly by quantum theory, while many interesting phenomena involve large numbers of atoms (in the range $10^{3}-10^{9}$ ), which quantum mechanical approaches cannot handlel. In this sense, silicon represents an ideal candidate for modeling by an empirical interatomic potential: the development of a potential will at once put to a rigorous test our understanding of the physics of covalent bonding and, if successful, will enable the simulation of important complex processes that involve large number of atoms.

Many attempts to construct an empirical model for in teratomic interactions in Si have already been reported 2 . Of all these models, the Stillinger and Weber (SW) 3 and the Tersoft 6 potentials are the most widely used 3 . The SW potential consists of two- and three-body terms and was fitted to experimental properties of the diamond cubic (DC) and molten phase of silicon 3 It has been usef, for exammle, to study lattice dynamicst, point defectos surfaces 10 , and the liquid and amorphous phases 312 [4].
The Terseff potential (its three versions usually referred to as $\mathrm{T} 1 \mathrm{E}, \mathrm{T} 2 \mathrm{E}$, and $\mathrm{T} 3 \mathrm{G}$ ) consists of many-body interactions included in a bond order term and was fitted to $a b$ initio results for several Si polytypes. It has been used to study lattice efrnamicst, thermomechanical properties 15 , poipt defect 30 , 6 , and the liquid and amorphous phases 6.14 .

Although the SW and Tersoff functional forms have enough flexibility to describe a number of different configurations, their transferability to a wide class of structures remains in question. Several models have attempted to improve the description of configurations far from equilibrium and far from the database used to construct the potential, by changing the functional form, using higher order (up to five-body) expansion terms, increasing the number of fitting parameters (fip to 36), or expanding the set of fitted structures17 20. Despite the increased complexity, these models have been able to improve the description of local configurations only selectively, and often at a considerable increase in computational cost. This suggests that a simple extension to more elaborate functional forms or larger databases does not necessarily provide better description of covalent bonding. Considering the lack of transferability of existing models, it is of interest to develop a model for silicon with the following ingredients: superior description of local structures and computationally efficient evaluations of the energy and interatomic forces. 
In this article we present a new empirical potential for silicon using a theoretically motivated functional form which emphasizes chemical and physical trends21, and which is determined by fitting to a fairly small $a b$ initio database with only 13 parameters. This potential represents a considerable improvement over existing models in describing local structures and extended defects. It provides a good description of point defects in the bulk, the concerted exchange path for self-diffusion, and elastic properties of bulk silicon. It also predicts core structures of partial dislocations in the glide set $\{111\}$ in excellent agreement with $a b$ initio results. Disordered structures and phase transitions are also well-described, particularly the amorphous phase, which is better modeled by dynamical simulations using our potential than by any empirical preparation method.

The article is organized as follows: The functional form is presented in detail in section II. The fitting of the model is discussed in section III, along with tests of transferability for bulk crystal structures, defects and activation complexes. The fitted potential is then used to calculate core properties of physically relevant dislocations in section IV and the structure of disordered phases in section $\mathrm{V}$.

\section{FUNCTIONAL FORM}

Here we describe the functional form of the new potential, which we call the Environment Dependent Interatomic Potential (EDIP) for bulk silicon, and refer the reader to the companion papen 21 for theoretical justification of all the terms. The energy of a configuration $\left\{\vec{R}_{i}\right\}$ is expressed as a sum over single-atom energies, $E=\sum_{i} E_{i}$, each containing two- and three-body terms,

$$
E_{i}=\sum_{j \neq i} V_{2}\left(R_{i j}, Z_{i}\right)+\sum_{j \neq i} \sum_{k \neq i, k>j} V_{3}\left(\vec{R}_{i j}, \vec{R}_{i k}, Z_{i}\right),
$$

where $V_{2}\left(R_{i j}, Z_{i}\right)$ is an interaction between atoms $i$ and $j$ representing pairwise bonds, and $V_{3}\left(\vec{R}_{i j}, \vec{R}_{i k}, Z_{i}\right)$ is the interaction between atoms $i, j$ and $k$ centered at atom $i$ representing angular forces. Both types of interactions depend on the local environment of atom $i$ through its effective coordination number, defined by,

$$
Z_{i}=\sum_{m \neq i} f\left(R_{i m}\right)
$$

where $f\left(R_{i m}\right)$ is a cutoff function that measures the contribution of neighbor $m$ to the coordination of atom $i$ in terms of the separation $R_{i m}$. The neighbor function, depicted in Fig. 1, is unity for $r<c$, with a gentle drop from 1 to 0 between $c$ and $a$, and is 0 for $r>a$ :

$$
f(r)= \begin{cases}1 & \text { if } r<c \\ \exp \left(\frac{\alpha}{1-x^{-3}}\right) & \text { if } c<r<a \\ 0 & \text { if } r>a\end{cases}
$$

where $x=\frac{(r-c)}{(a-c)}$. A neighbor of atom $i$ at a distance $r<c$ is considered a full neighbor, while the neighbors between $c$ and $a$ give only a partial contribution to $Z_{i}$. The cutoffs are constrained to reproduce the coordinations of important crystal structures, e. g. $Z_{i}=4$ for the diamond lattice.

The two-body term includes repulsive and attractive interactions,

$$
V_{2}(r, Z)=A\left[\left(\frac{B}{r}\right)^{\rho}-p(Z)\right] \exp \left(\frac{\sigma}{r-a}\right),
$$

which go to zero at the cutoff $r=a$ with all derivatives continuous. Although this choice is quite similar to the SW two-body term (and indeed reduces to it exactly for small distortions of the diamond lattice), the bond strength adapts to changes in the local atomic environment. The coordination dependence introduces an asymmetry, $V_{2}\left(R_{i j}, Z_{i}\right) \neq V_{2}\left(R_{j i}, Z_{j}\right)$, similar to the Tersoff potential in that the strength of the attractive force is controlled by a bond order function $p(Z)$ that depends on the local coordination. This dependence is motivated by theoretical calculations which have demonstrated the weakening of the attractive interaction and the correspopding increase in bond length for increasing coordination 21 23. These studies reveal a shoulder in the function $p(Z)$ at the ideal coordination $Z_{0}=4$, where the transition from covalent $\left(Z \leq Z_{0}\right)$ to metallic $\left(Z>Z_{0}\right)$ bonding occurs. This theoretical dependence can be accurately captured by a Gaussian function 21 ,

$$
p(Z)=\mathrm{e}^{-\beta Z^{2}} .
$$

Figure 2 shows the $V_{2}(r, Z)$ term for several coordinations, compared to the two-body part of the SW potential $V_{2}^{S W}(r)$. Note that the fitted $V_{2}(r, Z)$ resembles closely the inverted $a b$ initio pair petentials for silicon crystals with the samecoordination 23 , a feature built into the function form 21.

The three-body term contains radial and angular factors,

$$
V_{3}\left(\vec{R}_{i j}, \vec{R}_{i k}, Z_{i}\right)=g\left(R_{i j}\right) g\left(R_{i k}\right) h\left(l_{i j k}, Z_{i}\right)
$$

where $l_{i j k}=\cos \theta_{i j k}=\vec{R}_{i j} \cdot \vec{R}_{i k} / R_{i j} R_{i k}$. The radial function is chosen to have the SW form,

$$
g(r)=\exp \left(\frac{\gamma}{r-a}\right)
$$

and goes to zero smoothly at the cutoff distance $a$. The angular function $h(l, Z)$ is strongly dependent on the local coordination through two functions $\tau(Z)$ and $w(Z)$ that control the equilibrium angle and the strength of the interaction, respectively. Theoretical considerations lead us to postulate the following form 21: 


$$
h(l, Z)=H\left(\frac{l+\tau(Z)}{w(Z)}\right),
$$

where $H(x)$ is a generic function satisfying the constraints, $H(x)>0, H(0)=0, H^{\prime}(0)=0$ and $H^{\prime \prime}(0)>0$. Our specific choice is

$h(l, Z)=\lambda\left[\left(1-e^{-Q(Z)(l+\tau(Z))^{2}}\right)+\eta Q(Z)(l+\tau(Z))^{2}\right]$.

We make the choice $w(Z)^{-2}=Q(Z)=Q_{0} e^{-\mu Z}$ to control the strength of angular forces as function of coordination. The three-body angular function becomes flatter (and hence angular forces become weaker) as coordination increases, representing the transition from covalent bonding to metallic bonding. The angular function $h(l, Z)$ has two major contributions. The first, $H_{1}(x) \propto 1-\mathrm{e}^{-x^{2}}$, is symmetric about the minimum, becoming flat for small angles. This choice of shape for the angular function was also used by Mistriotis, Flyntzanis and Farantos (MFF)18, but due to its environmentdependence our angular function is fundamentally different. In a preliminary fitted version $\rightarrow$ f EDIP, we included only this flat, symmetric terme24, but we found that it is not suitable for several structures (particularly the amorphous phase). Indeed, a more asymmetrical angular function is suggested by approximations of quantum-mechanical (tight-binding) model 25202 apd exact inversions of $a b$ initio cohesive energy curves 23 , so the present form of EDIP also contains a second term, $H_{2}(x) \propto x^{2}$ (identical in shape to the SW form but containing environment-dependence), which gives a stronger interaction for small angles.

The function $\tau(Z)=-l_{0}(Z)=-\cos \left(\theta_{0}(Z)\right)$ controls the equilibrium angle $\theta_{0}(Z)$ of the three-body interaction as a function of coordination. This feature of the potential makes it possible to model the proper hybridization of atoms in different environment,21. 2 : If a silicon atom is three- or four-fold coordinated, it will prefer to form $s p^{2}$ or $s p^{3}$ hybrid bonds with equilibrium angles $\theta_{0}(3)=120^{\circ}$ and $\theta_{0}(4)=109.471^{\circ}$, respectively. For coordinations 2 and 6 , we take $\tau(2)=\tau(6)=0$ or $\theta_{0}(2)=\theta_{0}(6)=90^{\circ}$. For two-fold coordination, this choice describes the preference for bonding along two orthogonal $p$-states with the low-energy nonbonding $s$ state fully occupied. For six-fold coordination, the choice $\theta_{0}(6)=90^{\circ}$ reflects the $p$ character of the bonds (which are also metallic, as discussed below). We construct $\tau(Z)$ to interpolate smoothly between the special points $(Z=2,3,4,6)$ with the following form,

$$
\tau(Z)=u_{1}+u_{2}\left(u_{3} e^{-u_{4} Z}-e^{-2 u_{4} Z}\right),
$$

with the parameters chosen as $u_{1}=-0.165799, u_{2}=$ $32.557, u_{3}=0.286198$, and $u_{4}=0.66$, resulting in the curve shown in Fig. 3, which is consistent with the results of quantum-mechanical approximation 29 . Note that these parameters are theoretically determined and are not allowed to vary in the fitting described in the next section. Figure 4 shows the three-body term $V_{3}\left(\vec{R}_{i j}, \vec{R}_{i k}, Z_{i}\right)$ for three atoms at a distance 2.35 $\AA$, and for different coordinations. We also compare the three-body term to the SW three-body potential $V_{3}^{S W}\left(\vec{R}_{i j}, \vec{R}_{i k}\right)$. The SW angular form $h_{S W}\left(\theta_{j i k}\right)=$ $\lambda_{S W}\left(\cos \left(\theta_{j i k}\right)+1 / 3\right)^{2}$ penalizes the configurations with angles smaller than $90^{\circ}$ with a large positive contribution. In contrast, the angular function of our model potential gives a considerably weaker interaction at small angles.

In summary, this implementation of EDIP for bulk silicon has 13 adjustable parameters: $A, B, \rho, \beta, \sigma, a, c$, $\lambda, \eta, \gamma, Q_{0}, \mu$ and $\alpha$. It also has continuous first and second derivatives with respect to the atomic position vectors. The functional form already contains considerable information about chemical bonding in bulk silicon taken directly from theoretical studies, mostly of ideal crystal structures. The (relatively few) adjustable parameters provide the necessary freedom to extrapolate these bonding dependences for defect structures strictly outside the theoretical input, as described below.

We close this section by addressing the crucial issue of computational efficiency (which motivates the use of empirical methods in the first place). The environment terms in the two- and three-body iterations require extra loops in the force calculation. In the case of the threebody interaction, it requires a four-body loop, that would make a force evaluation more expensive than with the SW potential. However, the four-body loop needs to be performed only for those neighbors $l$ of atom $i$ in which $\partial f\left(R_{i l}\right) / \partial R_{i l} \neq 0$. This happens only when atoms are in the range $c<r<a$, i.e. only for a small number of the neighbors. Therefore, one force evaluation using our model is approximately as efficient as one using the SW potential, showing that increased sophistication and realism can be achieved with insignificant computational overhead. In fact, since the fitted cutoff distance (given in the next section) is smaller than the corresponding SW cutoff, computing forces with our model is typically faster than with the SW potential. For example, in liquid phase simulations on a Silicon Graphics R-10000 processor it takes $30 \mu \mathrm{sec} /$ atom to evaluate forces using EDIP, compared with $50 \mu \mathrm{sec} /$ atom using the SW potential.

\section{FITTING AND TESTS}

In order to determine the values of the adjustable parameters, we fit to a database that includes $a b$ initio result 30 , based on density functional theory in the local density approximation (DFT/LDA), for bulk properties (cohesive energy and lattice constant of the DC structure), selected valmes along the unrelaxed concerted exchange (CE) path 11 for self-diffusion, some formation energies of unrelaxed point defects (vacancy and interstitial at the tetrahedral and hexagonal configurations) 3234 , a few key values in the generalized stacking fault (GSF) en- 
ergy surface 35 , and the experimental elastic constant 36 . This rich set of configurations contains many of the important local structures found in condensed phases and bulk defects and thus improves the transferability of the model. The database does not include other high symmetry configurations, such as SC, BCC or FCC (as many of the existing empirical potentials have done), although the DC structure is required to be the lowest in energy. These hypothetical metallic structures have large enough energies compared to covalent ones to be considered irrelevant. Incidentally, fitting the model simultaneously to such a wide set of configurations and properties represents a considerable computational challenge. The fitting is accomplished using a least-squares approach, with each configuration in the database weighted appropriately. All parameter values are allowed to vary at once through a simulated annealing process. Theoretical estimates of the parameters were also used to restrict the range of parameter-space that needed to be explored21. Table [1 provides the best set of parameters we obtained.

The calculated energies and properties of several bulk structures as obtained from our potential and from $a b i n i-$ tio and other empirical potential calculations are given in Table [1. This compilation of results includes the DC structure which is the ground state of $\mathrm{Si}$, and several other high-coordination bulk structures. Although the latter structures were not included in the fitting database, our model provides a reasonable description of their energies and a good description of equilibrium lattice constants. Structures such as $\beta$-tin and BCT5 37 are also of interest because they have low-energy and relatively low-coordination ( 5 for BCT5 and 6 for $\beta$-tin) 2 . Experimentally, the DC phase transforms into the $\beta$-tin structure under pressure. For the $\beta$-tin structure, our model predicts a cohesive energy per atom higher than that of DC by $\Delta E=0.67 \mathrm{eV}$, and a lattice parameter $a_{o}=4.76 \AA$, as compared to $a b$ initio results of 0.21 $\mathrm{eV}$ and $4.73 \AA$ respectively. For the BCT5 structure, our model predicts $\Delta E=0.26 \mathrm{eV}$ and $a_{o}=3.36 \AA$, as compared to $a b$ initio results of $0.23 \mathrm{eV}$ and $3.32 \AA$, respectively.

Most existing empirical potentials give a poor (or marginally acceptable) description of elastic properties of the DC crystal, which directly affects the description of the crystal deformation. In the fitting database we included the three independent elastic constants $C_{11}, C_{12}$ and $C_{44}$ from experiment. Table III compares elastic constants, obtained with the homogeneous deformation method 38 , as given by our model with the results from other empirical potentials and with experimental results. The shear constant $C_{44}$ is particularly important for the description of long range interactionst. Although $C_{44}$ is underestimated by most empirical models, ours is reasonably close to the experimental value. Our potential also almost perfectly reproduces the ab initio value of the shear modulus $C_{4 \text { (t }}^{\circ}$ without internal relaxation $B$, as anticipated by theory 21. Table [II also includes other elastic properties, such as the second shear constant,
$C_{11}-C_{12}$, and the Cauchy discrepancy, $\Delta C=C_{12}-C_{44}$, both important for determining crystal stability 40 . By predicting a negative Cauchy discrepancy, our potential offers a qualitative improvement over most other exist ing potentialst and several tight-binding (TB) models 41 which give positive values. It also provides a quantitative improvement over other TB models that get the correct sign of $\Delta C$ 42 . In summary, our potential gives elastic constants in excellent agreement with experimental and $a b$ initio results. As we have shown in Ref.21, accurate elastic behavior is not simply the result of good fitting, but is rather a built-in feature of our functional form.

Point defects in the DC crystal involve large atomic relaxations and rebonding, thus representing the first test for the transferability of our model in describing local structures away from equilibrium. Our fitting database included the unrelaxed structures of the vacancy $(\mathrm{V})$ and the interstitial in tetrahedral $\left(\mathrm{I}_{T}\right)$ and hexagonal $\left(\mathrm{I}_{H}\right)$ configurations 30 . Table IV shows the formation energy for the unrelaxed and relaxed structures of $\mathrm{V}, \mathrm{I}_{T}$ and $\mathrm{I}_{\mathbb{H}}$ as obtained using our model, compared to ab initio 32 - 34 and TB calculations 41 , and from calculations 2 using the SW and Tersoff (T2 and T3) potentials. The relaxed structures are computed rsing an energy minimization conjugate-gradient method 43 . Although the SW and Tersoff potentials give a reasonable description of relaxed structures, they clearly fail in describing the energy released upon relaxation. In our model, on the other hand, the relaxation energies are much lower and in reasonably good agreement with ab initio calculations. We emphasize that none of the relaxation energies or structures were used in the fitting database. The $<110>$ split interstitial (the lowest energy interstitial configuration) is well described by our model, in spite of also not being included in the fitting. The formation energy for the relaxed structure of the $<110>$ split interstitial is 3.35 $\mathrm{eV}$ in our mpdel, compared to $3.30 \mathrm{eV}$ from ab initio calculations 44 , while the SW potential gives $4.68 \mathrm{eV}$.

The CE process 31 has been identified as a possible mechanism for self-diffusion in Si45. Most of the empirical potentials provide only a fair description of this important and complicated sequence of configurations 17 . Fig. 5 shows the energy for the unrelaxed CE path 17 from calculations based on DFT/LDAB0, the present model and the SW and Tersoff potentials. The results using our model agree reasonably well with those from DFT/LDA calculations, and are considerably better than those using the SW or Tersoff potentials. The activation energy for CE (an important quantity that enters in the calculation of diffusion rates) obtained from our model is 5.41 $\mathrm{eV}$, compared to $5.47 \mathrm{eV}$ from ab initio calculations, 7.90 $\mathrm{eV}$ from the $\mathrm{SW}$ potential, and $6.50 \mathrm{eV}$ from the Tersoff potential. The energy of the relaxed structure, which was not included in the fitting database, is $4.82 \mathrm{eV}$ using our model, in good agreement with the ab initio result of $4.60 \mathrm{eV}$.

The fitting database also included selected unrelaxed configurations of the generalized stacking fault (GSF) en- 
ergies. We considered three points for the the glide set and three for the shuffle set 35 of the $\{111\}$ glide plane. Table $\mathrm{V}$ shows the unstable stacking fault energy for the unrelaxed glide and shuffle $\{111\}$ planes as obtained from calculations using DFT/LDA35, SW, and the present interatomic potential. Our model gives good agreement with DFT/LDA calculations for the $<112>$ glide set, but underestimates the energy for the $<110\rangle$ glide set. Since our potential is short-ranged, it gives zero energy for the stable stacking fault, while the experimental value is $0.006 \mathrm{eV} / \AA^{2}$. The SW and Tersoff models, which are also short-ranged, give the same zero energy for the stable stacking fault. The TB model 14 , on the other hand, gives $0.005 \mathrm{eV} / \AA^{2}$, in agreement with DFT/LDA results. Nevertheless, given that the accuracy of empirical models is rarely better than a few tenths of an $\mathrm{eV}$ per atom, our vanishing stable stacking fault energy is not particularly problematic.

In summary, our potential provides an excellent description of configurations near equilibrium as well as a wide range of point defects in the DC structure. Although a number of these properties were explicitly fit, the superior description has been achieved with a small number of adjustable parameters that is greatly exceeded by the number of degrees of freedom inherent in these configurations. Thus, we suggest that it is our physically appropriate functional form rather than a flexible fitting strategy that has led to the improved description, a conclusion that is further supported by our results for extended defects and disordered phases discussed next.

\section{DISLOCATIONS}

A stringent test of the transferability of our model to local structures is obtained by calculating core properties of dislocations, about which no information was included in the fitting databass-Several $a b$ initid 4749 and tightbinding calculations 16 [0 have been performed for dislocations in silicon. Although such calculations are feasible only for small systems containing of order few hundred atoms, they provide important information, such as the core structure of the $90^{\circ}$-partial dislocation 14 , the kink structure in the $30^{\circ}$-partial dislocation 18 , and dislocation interactions 49 . However, full simulations of dislocation dynamics and its effect on the mechanical properties of the solid require much larger cells owing to the long range interaction of the stress fields, and therefore can only be performed using methods that are computationally less expensive. Empirical models have been used to study several aspects of dislocations in silicon 43 ,51,52. No such model has preven reliable enough to proyide a description of long range 51 as well as core properties 53 of dislocations at the same time. The flaw in describing long range interactions is due to a poor description of elastic forces 15 54, while the flaw in describing core preperties is due to a poor description of local structures 53 . Existing models do not give the correct structure for reconstructed cores: for example, the SW potential gives reconstruction only for the $30^{\circ}$-partial dislocation, and the Tersoff potential gives reconstruction only for the $90^{\circ}$-partial.

In the present study, all dislocation structures are computed ysing energy minimization methods 43 at constant stress 55 for a system of 3600 atoms, and the cell boundaries lie in the $[11 \overline{2}],[111]$, and $[110]$ directions. Fig. 6 shows (a) the unreconstructed and (b) the reconstructed core structure of a $90^{\circ}$-partial dislocation. The reconstruction energy is defined as the energy gain (per unit length) for the system to go from unreconstructed to reconstructed configuration. Table V1 compares the reconstruction energy in units of $\mathrm{eV} / b$ (where $b$ is the unit length of the dislocation, $b=3.84 \AA$ ), as given by our model and by SW, Tersoff, TB, and $a b$ initio calculations. Configuration (b) is neither stable nor metastable for the SW potential, i.e. the SW model does not support reconstruction for the $90^{\circ}$-partial dislocation 53 . The present model predicts configuration (b) as the lowest in energy, while configuration (a) is metastable. This model gives reconstruction energy of $0.84 \mathrm{eV} / b$, in excel lent agreement with the $a b$ initio value of $0.87 \mathrm{eV} / b$ 过. In our calculation, the recenstructed bonds are stretched by $2.1 \%$, while $a b$ initid 47 and TBE calculations give bonds stretched by $2.6 \%$ and $3.0 \%$ respectively.

Fig. 7 shows (a) the unreconstructed and (b) the reconstructed core structures of a $30^{\circ}$-partial dislocation. The results for the reconstruction energy from $a b$ initio calculations 56, and from empirical potential calculations using the SW, Tersoff and our model, are presented in table VI. The Tersoff potential gives negative reconstruction energy, so that the unreconstructed configuration (a) would be the more stable 33 , contrary to experimental and $a b$ initio results. Although the SW model gives the correct reconstructed configuration, the reconstruction energy is twice as large as the ab initio result. Our model, on the other hand, gives the reconstruction energy in very good agreement with the $a b$ initio calculation. We find that the reconstructed bonds in the dislocation core are stretched by $3.6 \%$. The ab initio calculations 56 give bond stretching of $4.2 \%$ at most.

It is important to mention that we found a few metastable partially reconstructed configurations between the unreconstructed and reconstructed configurations for both the $90^{\circ}$ - and $30^{\circ}$-partial dislocations. Such configurations are artifacts of our description of the local environment during changes incoordination, and probably have no physical meaning $\$$.

A defect in the core of a reconstructed dislocation is called an antiphase defect (APD) 配. Fig. 8 shows APD configurations in (a) a $30^{\circ}$-partial dislocation and (b) a $90^{\circ}$-partial dislocation. Table $\mathrm{NI}$ gives the corresponding APD formation energies. Since the SW model does not produce a reconstructed configuration for $90^{\circ}$-partial dislocations, there is no stable APD configuration for this case. For a $30^{\circ}$-partial dislocation, the SW model gives an APD formation energy much larger than $a b i n i-$ 
tio calculations 56. The Tersoff potential gives a negative value for the APD formation energy of the $30^{\circ}$-partial and a considerably smaller energy for the APD in a $90^{\circ}$ partial. Our model gives the APD formation energy in good agreement with ab initio calculations for the $30^{\circ}$ partial dislocation. For the $90^{\circ}$-partial dislocation, to our knowledge there is no ab initio APD energy calculation available, but our APD energy is somewhat low compared to TB calculations 50 .

From the above comparisons we have established that our empirical model is the first to give a full description of core properties of both the $90^{\circ}$ and $30^{\circ}$-partial dislocations in silicon with reasonable accuracy. Our model predicts reasonable reconstruction energies and provides a good description of local deformations in the dislocation cores. These results demonstrate remarkable transferability since the local atomic configurations present in dislocation cores are quite different from the structures included in the fitting database.

\section{DISORDERED STRUCTURES}

Another stringent test of the transferability of our model for bulk material is the calculation of structural properties of the liquid and amorphous phases. Such disordered structures contain a rich set of local bonding states from covalent (amorphous) to metallic (liquid) about which no information was included in our fitting procedure. Existing environment-independent potentials have had considerable difficulty in simultaneously describing the crystalline, liquid and amorphous phase 2, 4458 60. In the preceding sections we have demonstrated an improved description of the crystalline solid and its defects, so we now turn our attention to whether our environment-dependence can extrapolate these successes to the liquid and amorphous phases.

Liquid phase. The SW potential has been shown to reproduce the pair correlation function $g(r)$ of the liquid 14 . It also predicts the melting temperature $T_{m}$ to within a few hundred degrees of the experimental value of $1685 \mathrm{~K}$ (although it was explicitly fit to reproduce $T_{m} 3^{3}$. In spite of these successes, the $\mathrm{SW}$ potential has difficulty in describing the structure of liquid14, i.e. it does not reproduce the $a b$ initio bond angle distribution, overly favoring angles near tetrahedra 58 . The Tersoff potentials, on the other hand, predicta $g(r)$ that favors the unphysical four-fold coordination $\mathbf{3}$, and the only version predicting reasonable liquid structure is T36,14. The melting temperature, however, is greatly overestimated by $\mathrm{T} 3$ at around $3000 \mathrm{~K} 6.14$. The first-neighbor bond angles and coordination statistics are not well described by T3, although the statistics are improved by using an (arbitrarily) longer coordination cutoff beyond the first minimum of $g(r) 14$. We emphasize that the T2 potential, the mostsuccessful parameterization of the Tersoff model overalla, cannot decribe the liquid phase 5 .
We prepared a 1728-atom liquid sample with the present potential at $T=1800 \mathrm{~K}$ and zero pressure using standard simulation techniques, although we used a considerably lonore time and larger system size than in previous studies 61.62. The structural properties of the model liquid are shown in Fig. 9 and compared with the results of a 64-atom ab initio molecular dynamics study 58 (which are similiar to recent results with 343 atoms including electron spin effects and gradient corrections 63 ). The pair correlation function $g(r)$ shows excessive short-range order with our potential, as evidenced by the overly sharp first neighbor peak containing around 4.5 first neighbors, smaller than the experimental value of 6.4. This is consistent with the fact that in our model the density of the liquid is somewhat smaller than that of the solid, while in reality silicon becomes $10 \%$ more dense upon melting 64 . Although these features are unphysical, the present model offers a qualitative improvement in the bond angle distribution function $g_{3}\left(\theta, r_{m}\right)$, which gives the (normalized) distribution of angles between pairs of bonds shorter than $r_{m}$, the first minimum of $g(r)$. As shown in Fig. 9, our potential predicts the auxiliary maximum at $\theta=60^{\circ}$, although the primary maximum is shifted toward the tetrahedral angle away from the $a b$ initio most probable angle of $\theta=90^{\circ}$. The present model is the first to capture the bimedal shape of the first-neighbor bond angle distribution 65 .

The thermodynamic properties of the melting transition (aside from the change in density) are reasonably well-described by our model, in spite of its not having been fit to reproduce any such quantities. The bulk melting point $T_{m}$ predicted by our model is $1370 \pm 20 \mathrm{~K}$, which is $20 \%$ below the experimental value. The melting point was measured for a finite sample with $(100) 2 \times 1$ surfaces, heated from $300 \mathrm{~K}$ to $1500 \mathrm{~K}$ in 2 ns (over 10 million time steps). A bulk solid with periodic boundary conditions superheats and melts around $2200 \mathrm{~K}$. The latent heat of melting is $37.8 \mathrm{~kJ} / \mathrm{mol}$, in reasonable agrement with the experimental value of $50.7 \mathrm{~kJ} / \mathrm{mol}$, closer than the SW value of $31.4 \mathrm{~kJ} / \mathrm{mol}$.

In summary, although the liquid has some unphysical features with our potential, it offers some improvements, particularly in describing bond angles. It is important to emphasize that reasonable liquid properties are predicted by our model without any explicit fitting to the liquid phase; in contrast, the only two potentials reported to give an adequate description of the liquid, SW and MFF, were each fit to reproduce the melting point 318 . With our model, the reduced density and excess of covalent bonds may be artifacts of the short cutoff of our potential, which is appropriate for the covalently-bonded structures used in the fitting, but is perhaps too short to reproduce overcoordination in metallic phases like the liquid 66 .

Amorphous phase. Experimentally, amorphous silicon is known to form a random tetrahedral network, with long-range disfrder and short-range order similar to that of the crystal67,68. Ab initio molecular dynamics sim- 
ulations of quenching a 64-atom liquid predict almost $97 \%$ four-fold coordination 59 . An empirical potential would be invaluable in exploring larger system sizes and longer relaxation times than are feasible from first principles, but unfortunately no existing potential is capable of quenching directly from the liquid to the amorphous phase. Instead, empirical model liquids typically transform into glassy phases upen cegling, characterized by frozen-in liquid structure11 13,60.69. Alternatively, in the case of the $\mathrm{T} 2$ potential quenching results in a reasonable amorphous structure 5,16, but the original liquid phase is not realistic and already contains excessive tetraherdal order5. Therefore, it has been impossible to simulate an experimentally relevant math to the amorphous structure (e.g. laser quenching 70 ), and artificial preparation methods have been required to create large-scale amerphous structures with empirical potentials 11 13,60, 61,22 . For example, the so-called indirect SW amorphous (ISW) structure is created by increasing the strenoth of the SW three-body interaction during the quench 1113 . The ISW structure is stable with the unaltered SW potential, but since it has $81 \%$ four-fold coordinated atoms 11 , it only bears a weak resemblance to the real amorphous structure60. Abandoning molecular dynamics, an improved amorphous phase with close to $87 \%$ four-fold coordination can be generated using the bond-switching algorithm of Wooten, Winer and Weaire 71 , but such a method does not permit accurate simulation of atomic motion. A more realistic, large-scale amorphous structure can be prepared using a hybrid of these methods: Holender and Morgan create an amorphous structure of over $10^{5}$ atoms with almost $94 \%$ four-fold coordination by patching together a number of smaller WWW structures, thermally treating the sample at high temperatures and then relaxing it using molecular dynamics with the SW potential modified for stronger angular forces 72 . As these authors emphasize, however, this preparation method (which we call the HM2 model) was designed by trial-and-error to fit the experimental structure factor and bears no resemblance to the real experimental generation of a-Si. They also report that if the SW potential is not modified, their preparation method results in a structure (which we call the HM1 model) with only $74 \%$ four-fold coordination.

Remarkably, the present model predicts a quench directly from the liquid into a high-quality amorphous structure. The phase transition is quite robust, since it occurs even with fast cooling rates. For example, quenching at $-300 \mathrm{~K} / \mathrm{ps}$ leads to a reasonable structure with $84 \%$ four-fold coordination. At much slower quench rates of $-1 \mathrm{~K} / \mathrm{ps}$, an improved structure of 1728 atoms at $T=300$ $\mathrm{K}$ and zero pressure is produced with almost $95 \%$ fourfold coordination. The excess enthalpy of the amorphous phase compared with the crystal is $0.22 \mathrm{eV} /$ atom, closer to experimental values $\leq 0.19 \mathrm{eV} /$ atom 70 than the $a b$ initio value $0.28 \mathrm{eV} /$ atom (probably due to the constrained volume and small system size used in the $a b$ initio study 59 ).
The coordination statistics of the amorphous phase obtained with our model, given in Table VII, are closer to $a b$ initio results 59 than with most of the empirical models described above. The HM2 model provides a comparable description, but we stress that its preparation procedure is unphysical and that the modification of the SW potential necessary to achieve the improved description (see the difference between HM1 and HM2 in Table VII) degrades many important properties, such as elastic constants, defect formation energies and the melting point. Since realistic preparation methods and dynamics have not been achieved with interatomic potentials, in the following we compare our results only with experiments and $a b$ initio simulations.

The pair correlation function shown in Fig 10 (a) is in good agreement with ab initio results59. Moreover, Fig. 11 shows that the radial distribution function $t(r)=4 \pi \rho r g(r)$ is in excellent agreement with the results of neutron scattering experiments by Kugler et. al 68 (using their experimental density $\rho=0.054$ atoms $/ \AA^{3}$ for comparison). The persistence of intermediate-range order up to 10 Åcaptured by our model as in experiment is a strength of the empirical approach, since this distance is roughly the size of the periodic simulation box used in the $a b$ initio studies 59 . Given the limited resolution of the experimental data, especially at small $r$ (large $q$ in the structure factor), the sharper first three peaks with our model may be interpreted as refinements of the experimental results. In Table VIII we summarize a detailed comparison of features of a-Si as obtained with our model and from ab-initio results, against those revealed by experiment. Overall the agreement between experiment is very satisfactory, with the results of the present model somewhat closer to experimental values than $a b$ initio results as in the case of the enthalpy $\left(\Delta H_{a-c}\right)$ and the bond-length $\left(\sigma_{r_{1}}\right)$ and bond-angle $\left(\sigma_{\theta}\right)$ deviations.

The bond angle distribution $g_{3}\left(\theta, r_{m}\right)$ shown in Fig. 10 (b) is narrowly peaked just below the tetrahedral angle, and also reproduces the small, well-separated peak at $60^{\circ}$ observed in $a b$ initio simulations 59 (unlike in previous empirical models). The average angle is $108.7^{\circ}$ and standard deviation $13.6^{\circ}$ in close agreement with the experimental values 60 of $108.6 \pm 0.2^{\circ}$ and $10.2 \pm 0.8^{\circ}$ and $a b$ initio values 59 of $108.3^{\circ}$ and $15.5^{\circ}$, respectively. Notice that the peaks in both $g(r)$ and $g_{3}\left(\theta, r_{m}\right)$ are narrower and taller with our model than with $a b$ initio methods, which probably reflects the small system size and short times of the $a b$ initio simulations compared to ours. In summary, our potential reproduces the random tetrahedral network of amorphous silicon very well, following a realistic preparation procedure that starts with a liquid phase and cools it down without any artificial changes. 


\section{CONCLUSION}

We have developed a new empirical potential for silicon that provides a considerable improvement over existing models in describing local structures away from equilibrium. The model introduces a theoretically motivated functional form that incorporates several coordination dependent functions to adapt the interactions for different coordinations. The fitted potential faithfully reproduces the elastic constants of the equilibrium DC structure and also captures the energetics of a wide range of point and extended defects and related activation energies. The superior description of bulk phases and defects is achieved with only thirteen fitting parameters, indicating exceptional transferability of the EDIP functional form. Its extended range of success over existing models with comparable numbers of parameters cannot be attributed to fitting alone.

For dislocations, this is the first empirical model to give a full description of core properties. It predicts the correct reconstruction for both the $90^{\circ}$ - and $30^{\circ}$-partial dislocations, and the reconstruction energies are in agreement with $a b$ initio data. The bond stretching is also in good agreement with $a b$ initio results, pointing to the fact that this model predicts reasonably accurately not only the energies but also the local structure of dislocation cores.

This is the also the first empirical model to predict a quench directly from the liquid to the amorphous phase. The quality of the resulting amorphous phase, with almost $95 \%$ four-fold coordination, is better than with any existing empirical preparation method. In some ways the amorphous phase with our model is even somewhat closer to experiment than with $a b$ initio simulations (surely because the latter are limited to very small system sizes and very short times), which is an encouraging success of the empirical approach to materials modeling.

Our model possesses the same level of efficiency as the SW potential, and simulations involving thousands of atoms may be readily performed on typical workstations. Therefore, it holds promise for successful applications to several systems that are still inaccessible to $a b$ initio calculations and are outside the range of validity of other empirical models. Taking into account the success of our model in describing dislocation properties, we suggest that it may also provide a reasonable description of small-angle grain boundaries and other such extended bulk defects. Considering its success with the amorphous and crystalline phases, the model may also describe the a-c interface and solid-phase epitaxial growth.

\section{ACKNOWLEDGMENTS}

Partial support was provided to JFJ, VVB, and SY by the MRSEC Program of the National Science Foundation under award number DMR 94-00334. Partial support was provided to MZB by a Computational Science Graduate Fellowship from the Office of Scientific Computing of the US Department of Energy and by the Harvard MRSEC , which is funded by NSF grant number DMR94-00396. JFJ also acknowledges partial support from Brazilian Agency FAPESP.

${ }^{1}$ M. C. Payne, M. P. Teter, D. C. Allan, T. A. Arias, and J. D. Joannopoulos, Rev. Mod. Phys. 64, 1045 (1992).

${ }^{2}$ H. Balamane, T. Halicioglu, and W. A. Tiller, Phys. Rev. B 46, 2250 (1992).

${ }^{3}$ F. H. Stillinger and T. A. Weber, Phys. Rev. B 31, 5262 (1985).

${ }^{4}$ J. Tersoff, Phys. Rev. Lett. 56, 632 (1986).

${ }^{5}$ J. Tersoff, Phys. Rev. B 37, 6991 (1988).

${ }^{6}$ J. Tersoff, Phys. Rev. B 38, 9902 (1988).

${ }^{7}$ X. P. Li, G. Chen, P. B. Allen, and J. Q. Broughton, Phys. Rev. B 38, 3331 (1988).

${ }^{8}$ I. P. Batra, F. F. Abraham, and S. Ciraci, Phys. Rev. B 35, 9552 (1987).

${ }^{9}$ M. Nastar, V. V. Bulatov, and S. Yip, Phys. Rev. B 53, 13521 (1995).

10 T. W. Poon, S. Yip, P. S. Ho, and F. F. Abraham, Phys. Rev. Lett. 65, 2161 (1990).

${ }^{11}$ J. Q. Broughton and X. P. Li, Phys. Rev. B 35, 9120 (1987).

${ }^{12}$ W. D. Luedtke and U. Landman, Phys. Rev. B 37, 4656 (1988).

13 M. D. Kluge, J. R. Ray and A. Rahman, Phys. Rev. B 36, 4234 (1987).

${ }^{14}$ M. Ishimaru, K. Yoshida, and T. Motooka, Phys. Rev. B 53, 7176 (1996).

${ }^{15}$ L. J. Porter, S. Yip, M. Yamaguchi, H. Kaburaki, and M. Tang, J. Appl. Phys. 81, 96 (1997).

${ }^{16}$ P. C. Kelires and J. Tersoff, Phys. Rev. Lett. 61, 562 (1988).

17 E. Kaxiras and K. C. Pandey, Phys. Rev. B 38, 12736 (1988).

18 A. D. Mistriotis, N. Flytzanis, and S. C. Farantos, Phys. Rev. B 39, 1212 (1989).

19 B. C. Bolding and H. C. Andersen, Phys. Rev. B 41, 10568 (1990).

20 J. R. Chelikowsky, J. C. Phillips, M. Kamal, and M. Strauss, Phys. Rev. Lett. 62, 292 (1989); J. R. Chelikowsky and J. C. Phillips, Phys. Rev. B 41, 5735 (1990); J. R. Chelikowsky, K. M. Glassford, and J. C. Phillips, 44, 1538 (1991).

${ }^{21}$ M. Z. Bazant, E. Kaxiras, and J. F. Justo, Phys. Rev. B 56, 8542 (1997).

22 A. E. Carlsson, P. A. Fedders, and C. W. Myles, Phys. Rev. B 41, 1247 (1990).

${ }^{23}$ M. Z. Bazant and E. Kaxiras, Phys. Rev. Lett. 77, 4370 (1996); in Materials Theory, Simulations and Parallel Algorithms, edited by E. Kaxiras, J. Joannopoulos, P. Vashista, 
and R. Kalia (Mater. Res. Soc. Proc. 408, Pitsburgh, PA, 1996) pp. $79-84$.

24 J. F. Justo, M. Z. Bazant, E. Kaxiras, V. V. Bulatov, and S. Yip, to appear in Mater. Res. Soc. Proc. 469 (Materials Research Society, 1997).

${ }^{25}$ P. Alinaghian, S. R. Nishitani and D. G. Pettifor, Phil. Mag. B 69, 889 (1994);

${ }^{26}$ A. E. Carlsson, in Many-Atom Interactions in Solids, edited by R. M. Nieminen, M. J. Puska and M. J. Manninen (Springer Proc. in Phys. 48, Springer-Verlag, Berlin, 1990), pp. 257-263.

27 A. E. Carlsson, in Solid State Physics: Advances in Research and Applications, edited by H. Ehrenreich and D. Turnbull (Academic, New York, 1990), 43, pp. 1-91.

${ }^{28} \mathrm{~S}$. Ismail-Beigi and E. Kaxiras, private communication.

${ }^{29}$ If we assume $p_{\sigma}=p p \sigma /|s s \sigma| \approx Z-1$, then the leading order angular function in the bond order potential expansion 25 has a minimum given by $\tau_{\sigma}(Z) \approx 1 /(Z-1)$ in close agreement with our choice of $\tau(Z)$ for $Z \geq 3$. For $Z=2$, the bond order potential predicts a favored angle of $180^{\circ}$ rather than our choice of $90^{\circ}$, but since bulk structures rarely have $Z<3$ this difference is not relevant in the present context.

30 The fitting database consists of DFT/LDA total energies computed using a plane wave basis with at least a $12 \mathrm{Ry}$ cutoff and adequate sampling of the Brillouin zone for reciprocal space integrations. Supercells for the point defect calculations included 53-55 atoms, so that long range elastic relaxation energies, on the order of $0.01 \mathrm{eV}$, are ignored. Stacking fault calculations involved 12 atom supercells. For example, the formation energies for the unrelaxed defects are: $3.3 \mathrm{eV}$ for the vacancy, $3.7 \mathrm{eV}$ and $4.3 \mathrm{eV}$ for the tetrahedral and hexagonal interstitials, and $5.47 \mathrm{eV}$ for the concerted exchange saddle point configuration.

${ }^{31}$ K. C. Pandey, Phys. Rev. Lett. 57, 2287 (1986).

${ }^{32}$ Y. Bar-Yam and J. D. Joannopolous, Phys. Rev. Lett. 52, 1129 (1984).

33 P.J. Kelly and R. Car, Phys. Rev. B 45, 6543 (1992).

${ }^{34}$ H. Seong and L. J. Lewis, Phys. Rev. B 53, 9791 (1996).

${ }^{35}$ E. Kaxiras and M. S. Duesbery, Phys. Rev. Lett. 70, 3752 (1993).

${ }^{36}$ G. Simmons and H. Wang, Single Crystal Elastic Constants and Calculated Aggregate Properties: A Handbook (MIT, Cambridge, MA, 1971).

${ }^{37}$ E. Kaxiras and L. L. Boyer, Modeling Simul. Mater. Sci. Eng. 1, 91 (1992).

${ }^{38}$ D. C. Wallace, Thermodynamics of Crystals (Wiley, New York, 1972).

${ }^{39}$ O. H. Nielsen and R. M. Martin, Phys. Rev. B 32, 3792 (1985).

40 J. Wang, S. Yip, S. R. Phillpot, and D. Wolf, Phys. Rev. Lett. 71, 4182 (1993).

${ }^{41}$ N. Bernstein and E. Kaxiras, Phys. Rev. B 56, 10488 (1997).

${ }^{42}$ I. Kwon, R. Biswas, C. Z. Wang, K. M. Ho, and C. M. Soukoulis, Phys Rev. B 49, 7242 (1994).

${ }^{43}$ V. V. Bulatov, S. Yip, and A. S. Argon, Phil. Mag. A 72, 453 (1995).

${ }^{44}$ P. E. Blöchl, E. Smargiassi, R. Car, D. B. Laks, W. Andreoni, and S. T. Pantelides, Phys. Rev. Lett. 70, 2435 (1993).
${ }^{45}$ K.C. Pandey and E. Kaxiras, Phys. Rev. Lett. 66, 915 (1991); E. Kaxiras and K. C. Pandey, Phys. Rev. B 47, 1659 (1993); A. Antonelli, S. Ismail-Beigi, E. Kaxiras, and K. C. Pandey, Phys. Rev. B 53, 1310 (1996).

${ }^{46}$ L. B. Hansen, K. Stokbro, B. I. Lundqvist, K. W. Jacobsen, and D. M. Deaven, Phys. Rev. Lett. 75, 4444 (1995).

47 J. R. K. Bigger et. al., Phys. Rev. Lett. 69, 2224 (1992).

${ }^{48}$ Y. M. Huang, J. C. H. Spence, and O. F. Sankey, Phys. Rev. Lett. 74, 3392 (1995).

49 T. A. Arias and J. D. Joannopoulos, Phys. Rev. Lett. 73, 680 (1994).

${ }^{50}$ R. W. Nunes, J. Bennetto, and D. Vanderbilt, Phys. Rev. Lett. 77, 1516 (1996).

${ }^{51}$ U. Trinczek and H. Teichler, Phys. Sta. Sol. A 137, 577 (1993).

52 A. S. Nandedkar and J. Narayan, Phil. Mag. A 61, 873 (1990).

${ }^{53}$ M. S. Duesbery, B. Joos, and D. J. Michel, Phys. Rev. B 43, 5143 (1991).

${ }^{54}$ H. Teichler, in Polycrystalline Semiconductors, ed. J. H. Werner, H. J. Moller, and H. P. Strunk, Vol. 35 (Springer, New York, 1989), p. 25.

${ }^{55}$ M. Parrinello and A. Rahman, J. Appl. Phys. 52, 7182 (1981).

56 T. A. Arias, V. V. Bulatov, S. Yip, and A. S. Argon (to be published).

57 T. Suzuki, S. Takeuchi, and H. Yoshinaga, in Dislocation Dynamics and Plasticity (Springer, New York, 1991), p. 99.

${ }^{58}$ I. Štich, R. Car and M. Parinello, Phys. Rev. B 44, 4262 (1991); Phys. Rev. Lett. 63, 2240 (1989).

${ }^{59}$ I. Štich, R. Car, and M. Parrinello, Phys. Rev. B 44, 11092 (1991); R. Car and M. Parinello, Phys. Rev. Let. 60, 204 (1988).

${ }^{60}$ K. Ding and H. C. Andersen, Phys. Rev. B 34, 6987 (1986).

61 The equations of motion are integrated with the fifth order Gear predictor-corrector scheme. The temperature is controlled with intermittent velocity rescaling (every 250-500 time steps) and the pressure with the Anderson extended system method. Folling a lattice start at $T=4000 \mathrm{~K}$, the 1728-atom sample is cooled and equilibrated at $T=1800$ $\mathrm{K}$ for about $50 \mathrm{ps}$. Structural quantities are averaged in space and time (10 ps).

${ }^{62}$ M. P. Allen and D. J. Tildesley, Computer Simulation of Liquids (Clarendon Press, Oxford, 1987).

${ }^{63}$ I. Sttich, M. Parinello and J. M. Holender, Phys. Rev. Lett. 76, 2077 (1996).

${ }^{64}$ V. M. Glazov, S. N. Chizhevskaya and N. N. Glagoleva, Liquid Semiconductors (Plenum, New York, 1969).

${ }^{65}$ A bimodal bond angle disribution, similar to the present one, has been reported for the T3 potentiall4, but the authors found it necessary to arbitrarily extend the coordination cutoff beyond the first minimum of $g(r)$ to obtain such results. These simulations were also performed at very high temperature $(3000 \mathrm{~K})$ and constant volume (set to the volume of the experimental liquid, not the T3 liquid).

${ }^{66}$ Similar conclusions have been regarding the short cutoff of the Tersoff potentials 5.14.

67 J. Fortner and J. S. Lannin, Phys. Rev. B 39, 5527 (1989).

${ }^{68}$ S. Kugler, G. Molinar, G. Petö, E. Zsoldos, L. Rosta, A. Menelle and R. Bellissent, Phys. Rev. B 40, 8030 (1989). 
${ }^{69}$ R. Biswas, G. S. Grest and C. M. Soukoulis, Phys. Rev. B 36, 7437 (1987).

${ }^{70}$ S. Roorda, W. C. Sinke, J. M. Poate, D. C. Jacobson, S. Dierker, B. S. Dennis, D. J. Eaglesham, F. Spaepen and P. Fuoss, Phys. Rev. B 44, 3702 (1991).
${ }^{71}$ F. Wooten, K. Winer and D. Weaire, Phys. Rev. Lett. 54, 1392 (1985).

72 J. M. Holender and G. J. Morgan, J. Phys.: Condens. Matter 3, 7241 (1991); 3, 1947 (1991).

TABLE I. Values of the parameters that define the potential, obtained from a simulated annealing fit to the database described in the text.

\begin{tabular}{ccc}
\hline \hline$A=7.9821730 \mathrm{eV}$ & $B=1.5075463 \AA$ & $\rho=1.2085196$ \\
$a=3.1213820 \AA$ & $c=2.5609104 \AA$ & $\sigma=0.5774108 \AA$ \\
$\lambda=1.4533108 \mathrm{eV}$ & $\gamma=1.1247945 \AA$ & $\eta=0.2523244$ \\
$Q_{0}=312.1341346$ & $\mu=0.6966326$ & $\beta=0.0070975$ \\
$\alpha=3.1083847$ & & \\
\hline \hline
\end{tabular}

TABLE II. Energy and lattice parameters for high symmetry structures. Here we consider the ground-state diamond cubic (DC), face centered cubic (FCC), body centered cubic (BCC), simple cubic (SC) and hexagonal close-packed (HCP) crystals. For DC, the cohesive energy per atom $E_{c}^{D C}$ is given in $\mathrm{eV}$, while for the other crystals the difference of the cohesive energy $E_{c}$ from the ground state DC crystal, $\Delta E=E_{c}-E_{c}^{D C}$, is given. All lattice constants $a_{o}$ are for the conventional unit cells in $\AA$. For the hexagonal crystals we also give the $c / a$ ratios. We also compute the lattice constant and binding energy of an isolated hexagonal plane (HEX). For this comparison we use the SW potential with the mescaled cohesive energy for the ground state, as described in Ref.2.

\begin{tabular}{lcccccc}
\hline \hline & & DFT/LDA & EDIP & SW & T2 & T3 \\
\hline $\mathrm{DC}$ & $E$ & -4.65 & -4.650 & -4.63 & -4.63 & -4.63 \\
& $a_{o}$ & 5.43 & 5.430 & 5.431 & 5.431 & 5.432 \\
\hline $\mathrm{SC}$ & $\Delta E$ & 0.348 & 0.532 & 0.293 & 0.343 & 0.318 \\
& $a_{o}$ & 2.528 & 2.503 & 2.612 & 2.501 & 2.544 \\
\hline $\mathrm{BCC}$ & $\Delta E$ & 0.525 & 1.594 & 0.300 & 0.644 & 0.432 \\
& $a_{o}$ & 3.088 & 3.243 & 3.245 & 3.126 & 3.084 \\
\hline $\mathrm{FCC}$ & $\Delta E$ & 0.566 & 1.840 & 0.423 & 0.548 & 0.761 \\
& $a_{o}$ & 3.885 & 4.081 & 4.147 & 3.861 & 3.897 \\
\hline $\mathrm{HCP}$ & $\Delta E$ & 0.552 & 0.933 & 0.321 & 0.551 & 0.761 \\
& $a_{o}$ & 2.735 & 2.564 & 3.647 & 2.730 & 2.756 \\
& $c / a$ & 1.633 & 2.130 & 0.884 & 1.633 & 1.633 \\
\hline $\mathrm{HEX}$ & $\Delta E$ & 0.774 & 0.640 & 1.268 & & \\
& $a_{o}$ & 3.861 & 4.018 & 4.104 & & \\
\hline \hline
\end{tabular}


TABLE III. Elastic constants $C_{11}, C_{12}, C_{44}, C_{44}^{\circ}$ (without internal relaxation) and bulk modulus $B$ of the diamond cubic structure in Mbar, and the values of two combinations, $C_{11}-C_{12}$ and $C_{12}-C_{44}$, that are important for stability. The experimental values are from Ref. 36 and the tight-binding results from Ref 41 . The $a b$ initio result for $C_{44}^{\circ}$ (LDA) is from Ref.39.

\begin{tabular}{cccrcccc}
\hline \hline & EXPT & LDA & EDIP & SW & T2 & T3 & TB \\
\hline$C_{11}$ & 1.67 & & 1.75 & 1.61 & 1.27 & 1.43 & 1.45 \\
$C_{12}$ & 0.65 & & 0.62 & 0.82 & 0.86 & 0.75 & 0.85 \\
$C_{44}$ & 0.81 & & 0.71 & 0.60 & 0.10 & 0.69 & 0.53 \\
$C_{44}^{\circ}$ & & 1.11 & 1.12 & 1.17 & 0.92 & 1.19 & 1.35 \\
$B$ & 0.99 & & 0.99 & 1.08 & 0.98 & 0.98 & 1.05 \\
$C_{11}-C_{12}$ & 1.02 & & 1.13 & 0.79 & 0.41 & 0.68 & 0.60 \\
$C_{12}-C_{44}$ & -0.16 & & -0.09 & 0.22 & 0.76 & 0.06 & 0.32 \\
\hline \hline
\end{tabular}

TABLE IV. Ideal (unrelaxed) formation energies $E_{f}^{\text {ideal }}$ of point defects (in $\mathrm{eV}$ ) and relaxation energies $\Delta E=E_{f}^{\text {ideal }}-E_{f}^{\text {relaxed }}$ using a 54-tom unit cell. The ab initio (DFT/LBA) results are from Refs. 3034 and tight-binding results from Ref. 11 .

\begin{tabular}{lccccccc}
\hline \hline & & DFT/LDA & EDIP & SW & T2 & T3 & TB \\
\hline$V$ & $E_{f}$ & $3.3-4.3$ & 3.47 & 4.63 & 2.83 & 4.10 & 4.4 \\
& $\Delta E_{f}$ & $0.4-0.6$ & 0.25 & 1.81 & 0.02 & 0.40 & 1.2 \\
\hline$I_{T}$ & $E_{f}$ & $3.7-4.8$ & 6.15 & 12.21 & 5.85 & 6.92 & 4.5 \\
& $\Delta E_{f}$ & $0.1-0.2$ & 2.10 & 6.96 & 0.82 & 3.47 & 0.5 \\
\hline$I_{H}$ & $E_{f}$ & $4.3-5.0$ & 6.86 & 17.10 & 5.39 & 8.22 & 6.3 \\
& $\Delta E_{f}$ & $0.6-1.1$ & 2.70 & 10.15 & 1.72 & 3.61 & 1.3 \\
\hline$C E$ & $E_{f}$ & 5.47 & 5.41 & 7.90 & 6.50 & & 5.5 \\
& $\Delta E_{f}$ & 0.90 & 0.59 & 3.26 & & & 1.8 \\
\hline \hline
\end{tabular}

TABLE V. Unstable Stacking Fault energy (in $J / \mathrm{m}^{2}$ ) for the unrelaxed glide and-shuffle $\{111\}$ planes as obtained from calculations using DFT/LDA 35 , SW, and the present interatomic potential.

\begin{tabular}{llcrc}
\hline \hline & & DFT/LDA & EDIP & SW \\
\hline glide & $<112>$ & 2.51 & 3.24 & 4.78 \\
& $<110>$ & 24.71 & 13.45 & 26.17 \\
\hline shuffle & $<110>$ & 1.84 & 2.16 & 1.38 \\
\hline \hline
\end{tabular}


TABLE VI. Reconstruction energy (in $\mathrm{eV} / b$ ) and APD energy (in $\mathrm{eV}$ ) for core structures of partial dislocations, where $b$ is the repeat distance of the dislocation. The DFT/LDA result for reconstruction of the $90^{\circ}$-partial dislocation is from Ref.47, and for the $30^{\circ}$-partial dislocation is from Ref56. Results for the interatomic potential calculations (SW and T2) are from Ref53. Tight-binding result for the $90^{\circ}$-partial dislocation is from Ref 50 .

\begin{tabular}{cccccc}
\hline \hline & DFT/LDA & EDIP & SW & T2 & TB \\
\hline Reconstruction & & & & & \\
$90^{\circ}$-partial & 0.87 & 0.84 & - & 0.37 & 0.68 \\
$30^{\circ}$-partial & 0.43 & 0.33 & 0.81 & -0.13 & \\
\hline APD & & & & & \\
$90^{\circ}$-partial & - & 0.41 & - & 0.37 & 1.31 \\
$30^{\circ}$-partial & 0.43 & 0.34 & 0.84 & -0.13 & \\
\hline \hline
\end{tabular}

\begin{tabular}{lrrrrrr}
\hline \hline & \multicolumn{2}{c}{ Direct Quench } & \multicolumn{4}{c}{ Artificial Preparation } \\
& LDA & EDIP & HM2 & WWW & ISW & HM1 \\
\hline$N_{3}$ & 0.2 & 0.23 & 2.18 & 1.2 & 0.0 & 0.0 \\
$N_{4}$ & 96.6 & 94.43 & 93.74 & 86.6 & 81.0 & 73.6 \\
$N_{5}$ & 3.2 & 5.34 & 4.04 & 11.8 & 18.1 & 24.6 \\
$N_{6}$ & 0.0 & 0.00 & 0.01 & 0.2 & 0.9 & 1.5 \\
\hline \hline
\end{tabular}

TABLE VII. Coordination statistics for model amorphous structures at room temperature. $N_{n}$ is the percentage of atoms with $n$ neighbors closer than the first minimum of $g(r)$. We compare structures generated by molecular dynamics simplation of a direct quench from the liquid using an ab initio method 59 (LDA) and our interatomic potential (EDIP) with structures generated by rarious artif ical preparation methods described in the text: HM2 72 , WWW 71 , ISW 11 and HM172.

\begin{tabular}{lccccccccc}
\hline \hline & $\rho_{a}$ & $\Delta H_{a-c}$ & $\bar{Z}$ & $\bar{r}_{1}$ & $\sigma_{r_{1}}$ & $\bar{r}_{2}$ & $\bar{r}_{3}$ & $\bar{\theta}$ & $\sigma_{\theta}$ \\
\hline EDIP & 0.04836 & 0.22 & 4.054 & 2.39 & 0.034 & 3.84 & 5.83 & 108.6 & 14.0 \\
EXPT & $0.044-0.054$ & $<0.19$ & $3.90-3.97$ & $2.34-2.36$ & $0.07-0.11$ & 3.84 & 5.86 & 108.6 & $9.4-11.0$ \\
LDA & - & 0.28 & 4.03 & 2.38 & 0.079 & 3.84 & - & 108.3 & 15.5 \\
\hline \hline
\end{tabular}

TABLE VIII. Comparison of thermodynamic and structurapperties of the present model (EDIP) for a-Si with (annealed) $a b$ initid59 and with (annealed) experimental 59.68.67, 00 results. Shown are the density $\rho_{a}$ in $\AA^{-3}$, the excess enthalpy $\Delta H_{a-c}$ compared to the crystal in eV/atom, the average coordination $\bar{Z}$, the mean $\bar{r}_{1}$ and standard deviation $\sigma_{r_{1}}$ of the first neighbor distance in $\AA$, the mean second $\bar{r}_{2}$ and third $\bar{r}_{3}$ neighbor distances in Aand the mean $\bar{\theta}$ and standard deviation $\sigma_{\theta}$ of the first neighbor bond angles in degrees. 


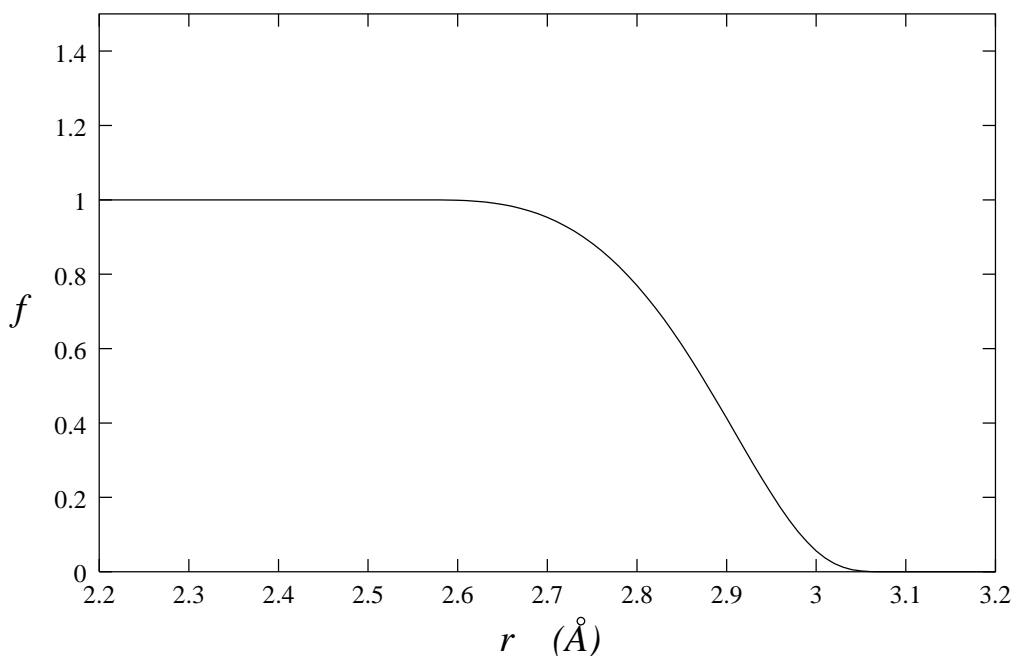

FIG. 1. The function $f(r)$ that determines the contribution of each neighbor to the effective coordination $Z$.

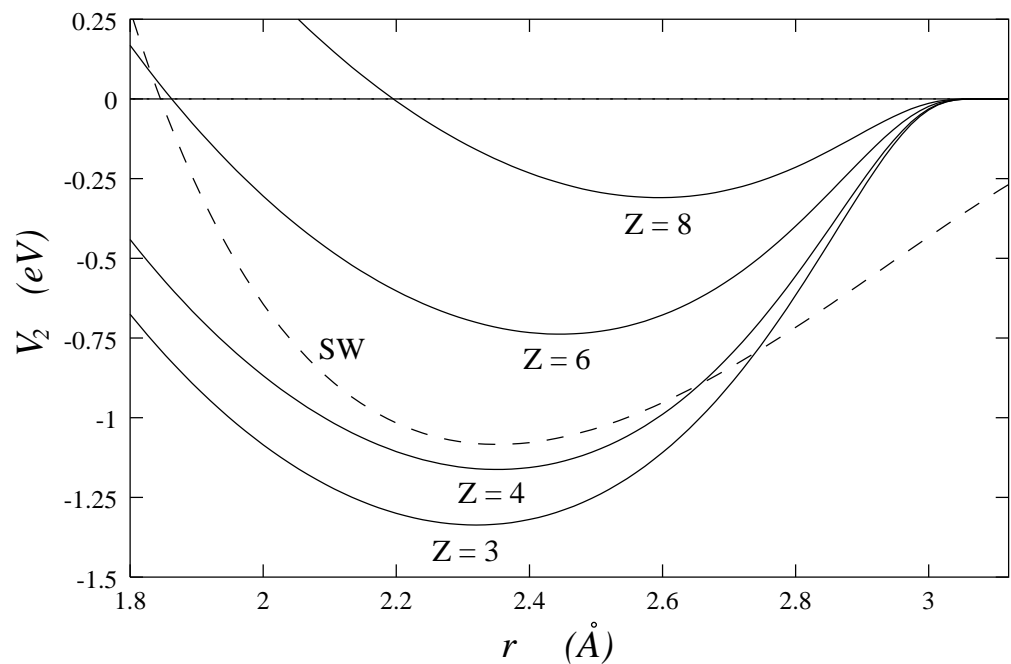

FIG. 2. The two-body interaction $V_{2}(r, Z)$ as a function of separation $r$ shown for coordinations 3, 4, 6 and 8 and compared with the Stillinger-Weber (SW) pair interaction. 


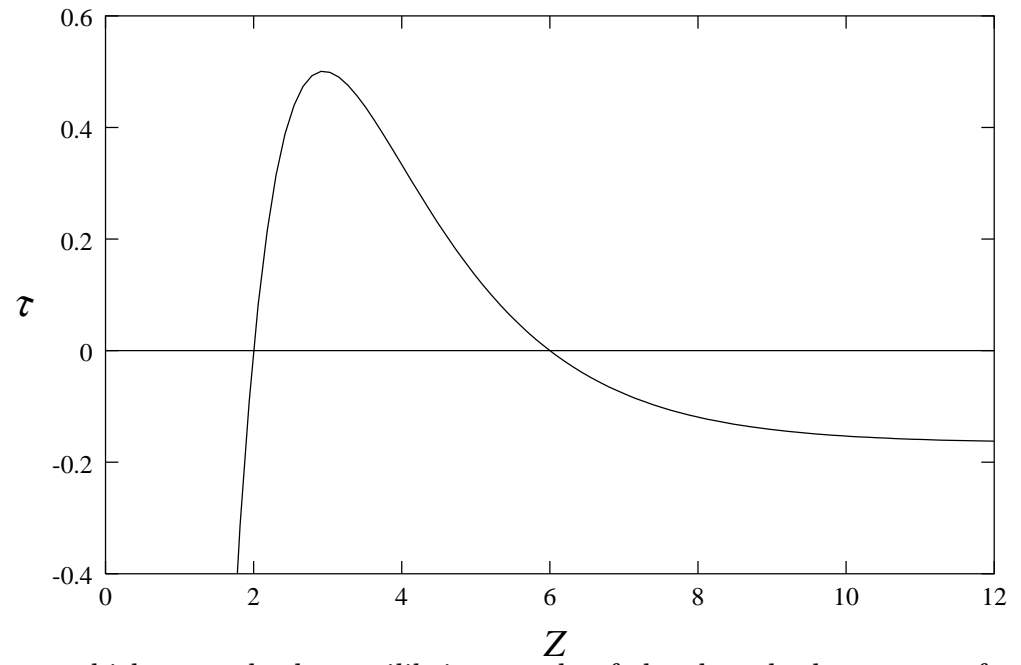

FIG. 3. $\tau$, which controls the equilibrium angle of the three-body term, as function of coordination.

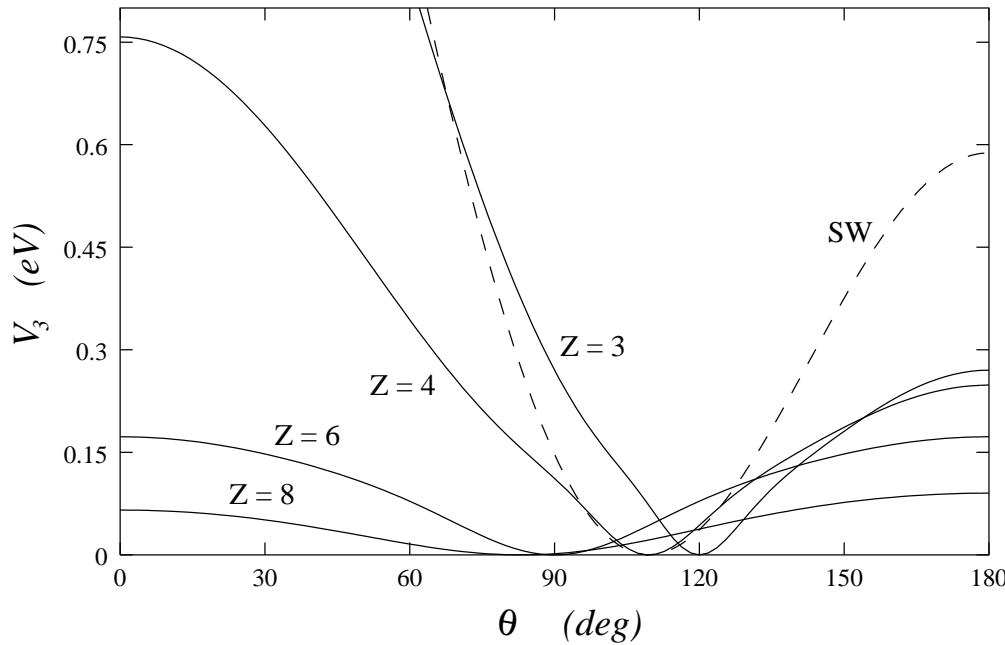

FIG. 4. The three-body interaction $V_{3}(r, r, \cos \theta, Z)$ for a pair of bonds of fixed length $r=2.35 \AA$ subtending an angle $\theta$, shown for coordinations 3, 4, 6 and 8 and compared with the Stillinger-Weber three-body interaction. 


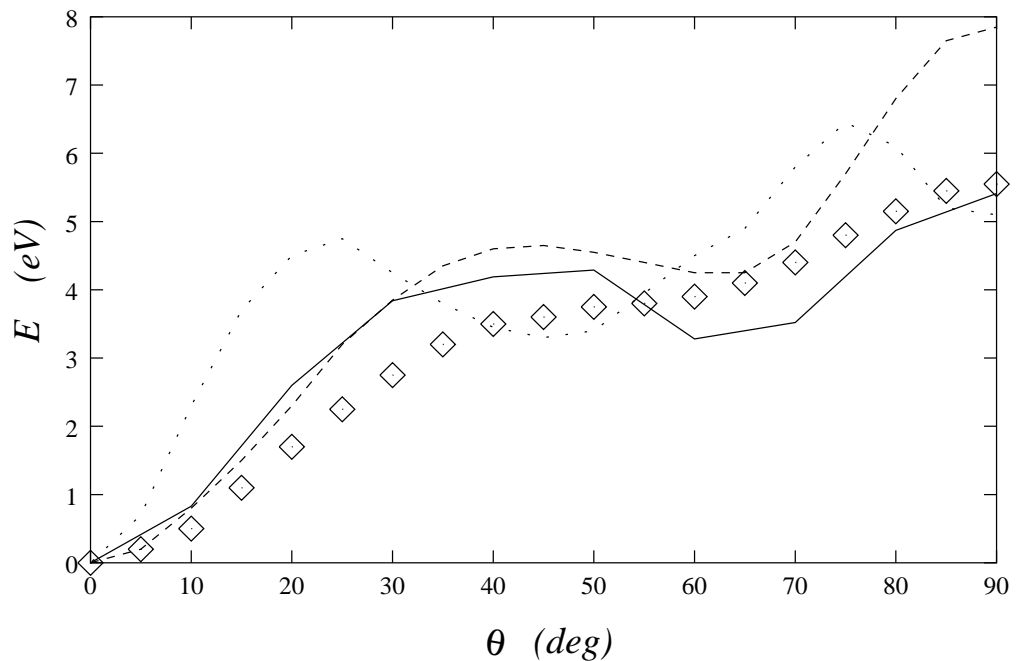

FIG. 5. Energy of the concerted exchange path obtained from calculations using DFT/LDA3d,B1 (diamonds), the SW (dashed line), the Tersoff (dotted line), and the present interatomic potential (solid line). The results from ab initio, SW and Tersoff are from Ref. 17 .

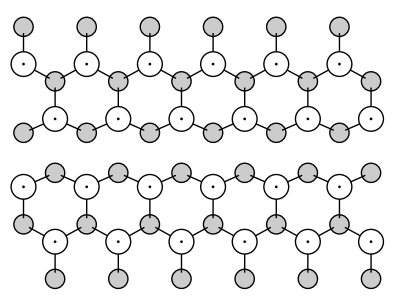

(a)

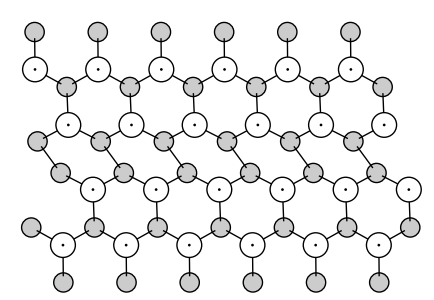

(b)

FIG. 6. Atomic structure in the core of a $90^{\circ}$-partial dislocation on the (111) plane. (a) Symmetric and (b) asymmetric reconstructions. Open and shaded circles represent atoms in two different (111) planes.

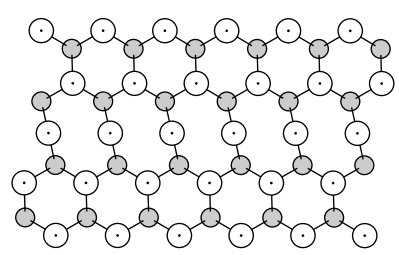

(a)

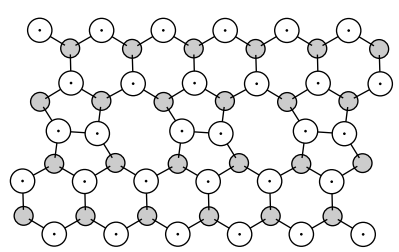

(b)

FIG. 7. Same as Fig. 6 for a $30^{\circ}$-partial dislocation. (a) Unreconstructed and (b) reconstructed configurations. 


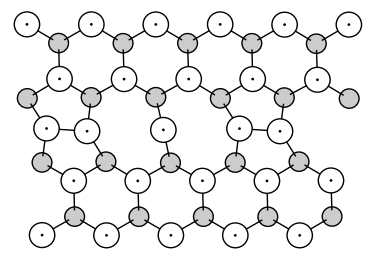

(a)

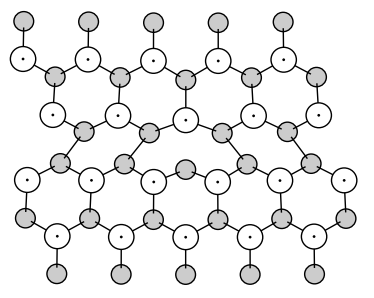

(b)

FIG. 8. Anti-phase defects (APD) for (a) the $30^{\circ}$-partial and (b) the $90^{\circ}$-partial dislocations.
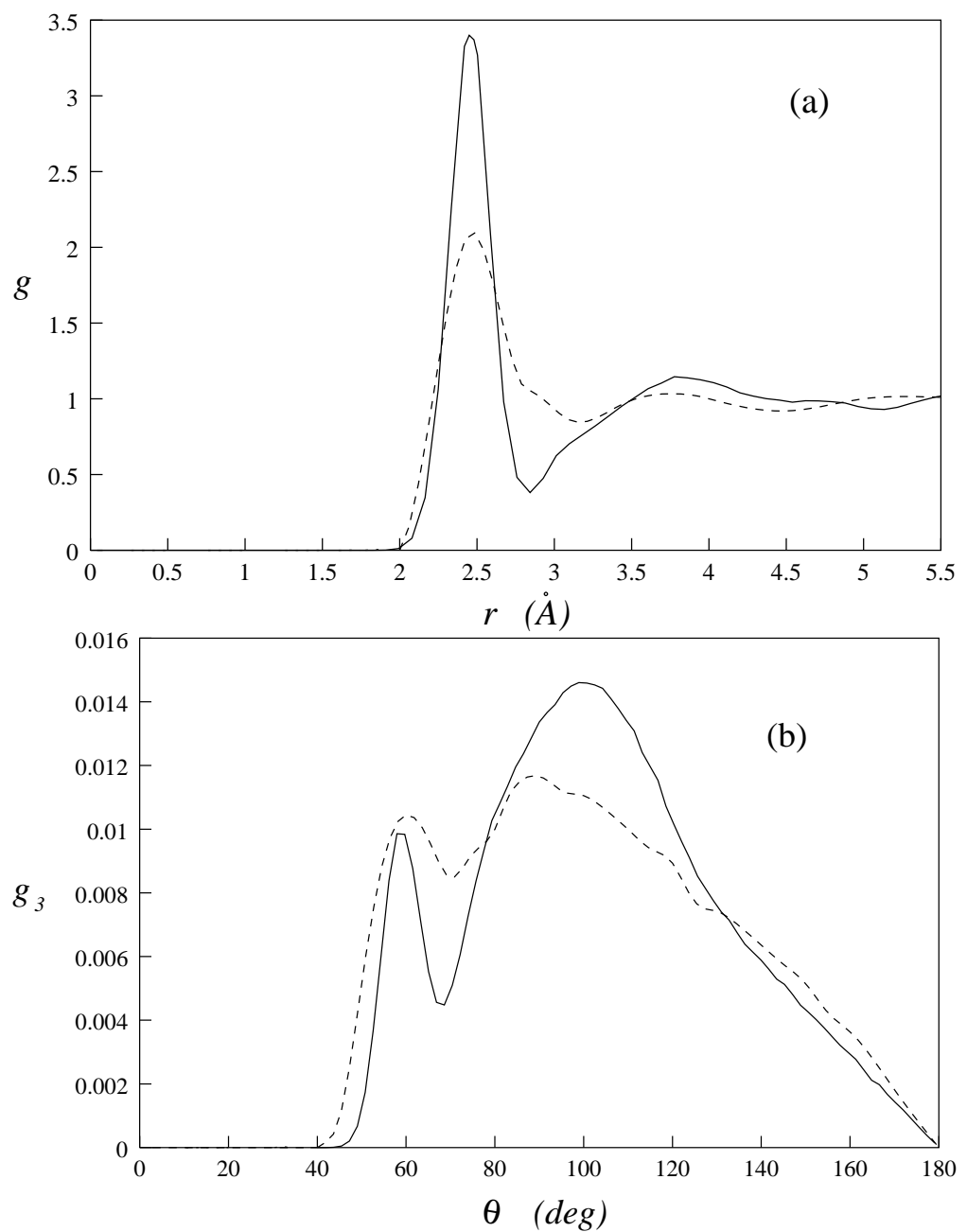

FIG. 9. (a) Pair correlation function and (b) bond angle distribution for the liquid at $T=1800 \mathrm{~K}$ and zero pressure with the present potential (solid lines) and the $a b$ initio model of Ref 58 (dashed lines). 

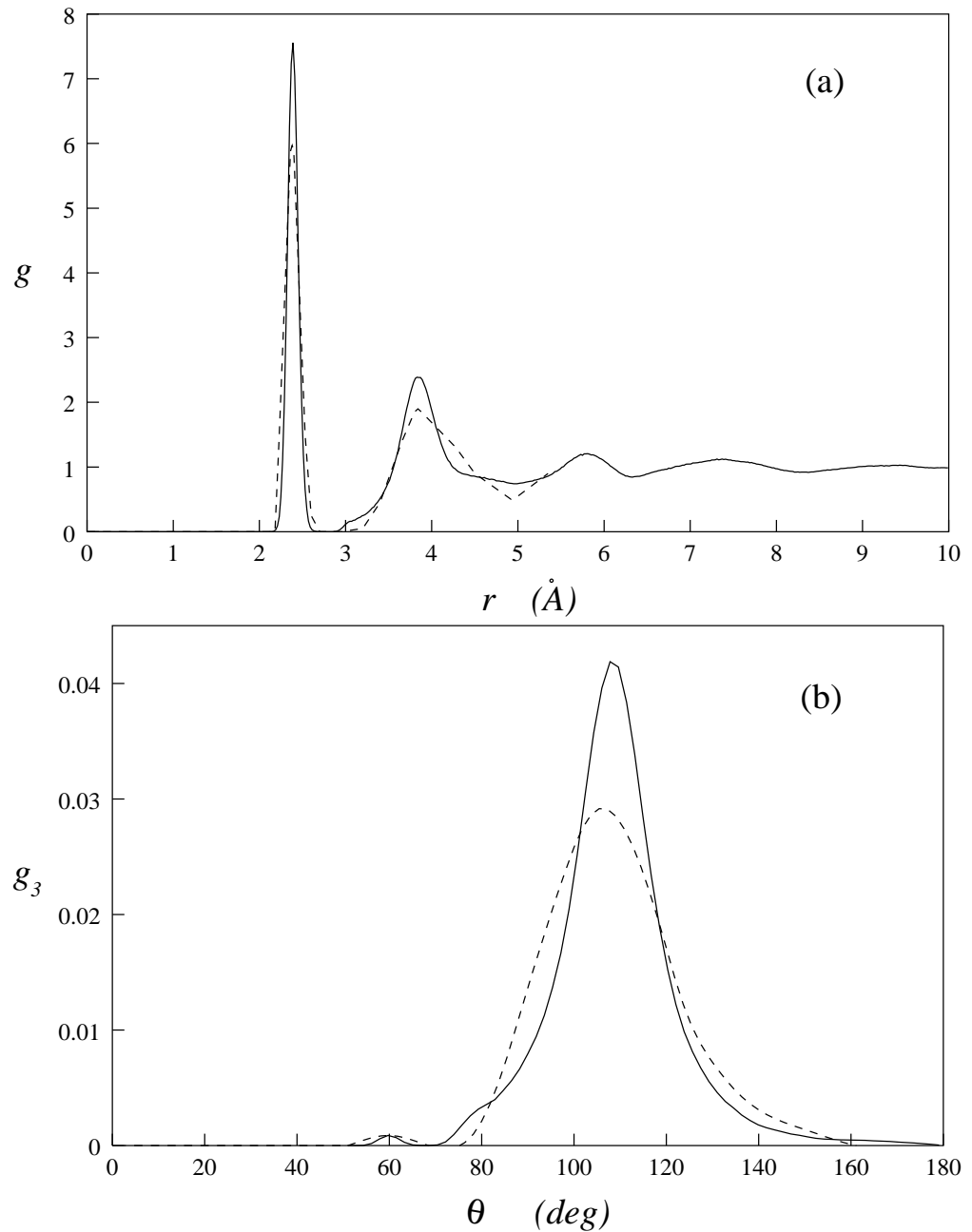

FIG. 10. (a) Pair correlation function and (b) bond angle distribution for the amorphous phase at $T=300 \mathrm{~K}$ and zero pressure with the present potential (solid lines) and the $a b$ initio model of Ref.59 (dashed lines).

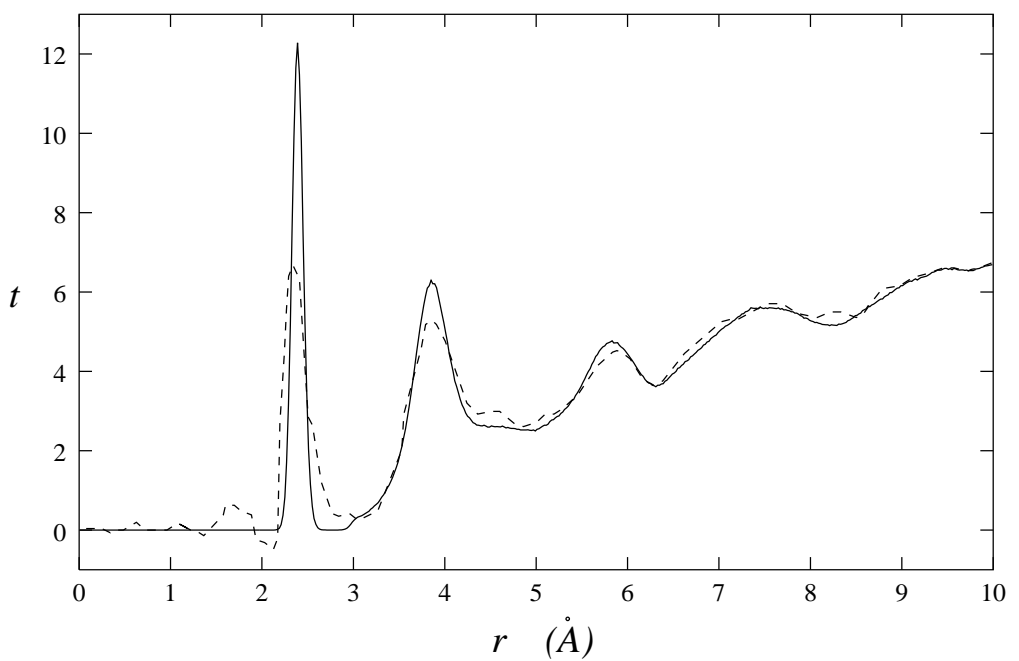

FIG. 11. Radial distribution function $t(r)=4 \pi \rho r g(r)$ for the amorphous phase at room temperature and zero pressure using our model, compared with the results of neutron scattering experiments on pure evaporated-beam-deposited a-Si thin films by Kugler et. al. 68 . 